\title{
Human resources for health: lessons from the cholera outbreak in Papua New Guinea
}

\author{
Alexander Rosewell, ab Sibauk Bieb, ${ }^{c}$ Geoff Clark, ${ }^{a}$ Geoff Miller, ${ }^{c}$ Raina Maclntyre ${ }^{b}$ and Anthony Zwi ${ }^{b}$ \\ Correspondence to Alexander Rosewell (e-mail: arosewell@yahoo.com)
}

Issue: Papua New Guinea is striving to achieve the minimum core requirements under the International Health Regulations in surveillance and outbreak response, and has experienced challenges in the availability and distribution of health professionals.

Context: Since mid-2009, a large cholera outbreak spread across lowland regions of the country and has been associated with more than 15500 notifications at a case fatality ratio of $3.2 \%$. The outbreak placed significant pressure on clinical and public health services.

Action: We describe some of the challenges to cholera preparedness and response in this human resource-limited setting, the strategies used to ensure effective cholera management and lessons learnt.

Outcome: Cholera task forces were useful to establish a clear system of leadership and accountability for cholera outbreak response and ensure efficiencies in each technical area. Cholera outbreak preparedness and response was strongest when human resource and health systems functioned well before the outbreak. Communication relied on coordination of existing networks and methods for empowering local leaders and villagers to modify behaviours of the population.

Discussion: In line with the national health emergencies plan, the successes of human resource strategies during the cholera outbreak should be built upon through emergency exercises, especially in non-affected provinces. Population needs for all public health professionals involved in health emergency preparedness and response should be mapped, and planning should be implemented to increase the numbers in relevant areas. Human resource planning should be integrated with health emergency planning. It is essential to maintain and strengthen the human resource capacities and experiences gained during the cholera outbreak to ensure a more effective response to the next health emergency.

\section{ISSUE}

Papua New Guinea is strengthening its capacity to identify, assess and respond to health emergencies in line with requirements of the International Health Regulations (IHR). ${ }^{1}$ To support the implementation of IHR (2005), the country has adopted components of the Asia Pacific Strategy for Emerging Diseases (2010), ${ }^{2}$ which outlines areas of achievement relating to health emergencies. Key to this strategy is the development of a national health emergencies plan that has been recently drafted by health authorities. To achieve the objectives of the national health emergencies plan, capable public health professionals are needed for the timely, effective response to public health emergencies at national and subnational levels.

\section{CONTEXT}

Papua New Guinea has the highest gross domestic product of the Pacific island countries, yet it invests only a small percentage (3.6\%) in health. ${ }^{3}$ As a consequence, the number of health care workers falls well short of internationally recommended staff-to-population ratios. The health workforce is not distributed according to the needs of the population; most (87\%) of the population live in rural areas, yet over half (52\%) of the health workers are in urban areas. ${ }^{4}$ Further human resource issues include weak standards of patient care, unhealthy workplace practices, run-down and inadequate infrastructure and equipment, and education and training that may not always meet the needs of the health care system. ${ }^{5}$ The tertiary education

\footnotetext{
World Health Organization, Port Moresby, Papua New Guinea

School of Public Health and Community Medicine, Faculty of Medicine, University of New South Wales, Sydney, New South Wales, Australia

National Department of Health, Port Moresby, Papua New Guinea.

Capacity Building Service Centre, Papua New Guinea.

Note: This article is based on a project first reported by the Human Resources for Health Knowledge Hub [Rosewell, A 2013, Human resources for health: practice and policy implications for emergency response arising from the cholera outbreak in Papua New Guinea, Human Resources for Health Knowledge Hub, Sydney, Australia].

Submitted: 22 April 2013; Published: 3 July 2013

doi: 10.5365/wpsar.2013.4.2.006
} 
system is currently unable to produce enough quality health workers. ${ }^{4}$

When a health crisis such as a cholera outbreak occurs, strategies for health workforce preparedness are crucial and must be in place to limit outbreakassociated morbidity and mortality. Human resource strategies should address the distribution of workers to rural areas, supervision, teamwork, remuneration and conditions for rural health workers. When cholera emerged in July $2009,{ }^{6}$ it caused widespread morbidity and mortality due in part to a lack of health system access and preparedness. In excess of 15500 cases were reported with a case fatality ratio of $3.2 \%$. No strategy was in place to address the supply of clinical or allied health workers. The subsequent spread of the disease to neighbouring provinces not only provided significant challenges to health authorities, ${ }^{7}$ but it also provided an opportunity to implement and evaluate novel human resource strategies. The purpose of this report is to outline the lessons learnt to improve management of human resources in future health emergencies.

\section{ACTION}

A qualitative approach was used to review human resource strategies during the cholera outbreak. Datagathering methods included document review of situation reports; key informant interviews with provincial cholera coordinators and members of cholera task forces at all levels, including governmental and nongovernmental stakeholders; and field observation. Specifically, key informant discussions were held with one provincial cholera coordinator, one Head of Mission and one Medical Coordinator for Médecins Sans Frontières (MSF Holland), three cholera experts from the World Health Organization and one adviser to a Provincial Health Adviser from a Provincial Health Office. This strategy was developed by the Human Resources for Health Knowledge Hub team based on their field research experience.

\section{OUTCOME}

\section{(1) Task forces are effective for outbreak management}

Working together, the National Cholera Task Force and Provincial Cholera Task Forces established a clear system of leadership and accountability for cholera outbreak response in each sector, enabled the National Department of Health to demonstrate its overall leadership and provided a framework for effective partnerships among international and national humanitarian actors in each sector at all levels of government. In provinces where there was a good working relationship between the Provincial Health Office and provincial hospitals before the outbreak, coordination generally functioned much better than in provinces where the relationship was poor. In provinces where the Provincial Health Office and provincial hospitals did not work cohesively before the outbreak, collaboration became exacerbated during the crisis, especially in the absence of good leadership. Through task forces, policy issues were identified and then moved forward through existing systems within health authorities.

\section{(2) Prioritizing interventions is crucial following risk assessment}

The process of conducting risk assessments and the subsequent prioritization of public health measures are crucial for effectively managing health emergencies, especially in the context of concurrent emergencies. Greater capacity to respond to health emergencies would be enabled by increasing staffing levels in relevant areas of health emergencies. The current staff numbers at all levels of government are vastly inadequate for running systems that generate information for risk assessment of health emergencies in Papua New Guinea. ${ }^{4}$ For example, without additional staff who can support provincial disease control officers with data management, ongoing surveillance, outbreak detection and verification processes between or during health emergencies risk assessment will remain challenged and prioritization of interventions may be based on scant information.

\section{(3) External staff can effectively coordinate outbreak response}

Two main models of subnational cholera task force coordination were adopted: (1) the cholera task force coordinator was the Provincial Health Adviser, and (2) the coordinator was a respected, effective leader from outside the government system. This flexibility in the subnational coordination modality was useful, as both models of coordination worked effectively. In the more challenging settings, recruiting coordinators from outside the provincial government system was successful. The Provincial Disaster Coordinator is not always the most appropriate coordinator of a health emergency. 
Task forces with active multisectoral participation were most effective.

\section{(4) Designated emergency response funds are essential at all levels}

Provinces that maintained a designated emergency response fund that could be immediately accessed were able to quickly implement control measures such as mobilizing rapid response teams. The emergency response budget must be able to cover the travel costs of relocated staff and their allowances. The financial accounts of Provincial Health Offices must be acquitted to ensure that task forces have control of the funding allocated for health emergencies and can use it for interventions that they have prioritized.

\section{(5) Local leaders are important for behaviour change}

It was noted on several occasions that the behaviour changes required of a community during cholera outbreaks are difficult to achieve, even if only required for a few months while the outbreak is occurring in a given setting. Communities are more likely to adopt recommended behaviours following repeated visits and messaging from respected persons such as village leaders, ward councillors, health workers or those organized by such leaders. Anecdotally, one-off visits by people with public address systems instructing the population what they should do did not appear to change behaviours during the period of the outbreak. When health authorities try to achieve behaviour change results without involving the community, the human resource burden is beyond the scope of their capacity. Behaviour change messages and materials required standardization, which was best achieved from the central level. The development of these tools could have been timelier and a pre-existing repository of communication tools would have been useful.

\section{(6) Timely recruitment of laboratory management is key to functionality}

National cholera surveillance worked effectively with only one functioning laboratory in Papua New Guinea. However, the vacant Director position at Central Public Health Laboratories could impact significantly the overall laboratory function and the capacity to take on new work (e.g. cholera surveillance) during crises. Re-establishing diagnostic capacity during outbreaks worked well at the provincial level; however, further support will be necessary to ensure sustainability of the training.

\section{(7) Effective surveillance systems rely on adequate numbers of trained staff}

Timely surveillance of outbreaks is a realistic goal in Papua New Guinea, but it is reliant on adequate staffing. During the cholera outbreak, the command and control centres facilitated the information management component of the health emergency. Given the limited staff and data management capacity at the subnational level, the national surveillance staff were frequently required to perform provincial data entry.

(8) Surveillance requires data management support but temporary workers are not sustainable

Supportive visits to the provinces, where data entry may have occurred for the first time, were sometimes the only way to stimulate the flow of data to the national level. Data managers who were recruited to work temporarily under the disease control officer during the cholera outbreak were crucial at the time of the emergency. However, because the situation continued for several months, when the temporary workers returned to their original positions, it took some time to replace them. Consideration should be given to having a permanent data manager position at the provincial level. If data managers were available between outbreaks, they could support a weekly reporting system for syndromes of public health importance. In provinces where there were challenges with the flow of cholera surveillance data, sometimes large amounts of data were never forwarded to the national level.

\section{(9) Formalizing the rapid response team was simple and effective}

Once the Senior Executive Management of the National Department of Health decided to formalize the national rapid response team, action was swiftly taken. Within weeks, the national rapid response team had completed its first investigation, confirming cholera and micronutrient deficiencies associated with high mortality among internally displaced persons. In addition to the technical support provided in field epidemiology, assistance was provided to provincial authorities in 
outbreak communication and water, sanitation and hygiene.

\section{(10) Leadership and training enable effective staff rotation policies}

In the context of inflexible systems for recruiting clinical staff for outbreak surge capacity, the rotation of district staff appeared to be a successful strategy for ensuring adequate case management, training staff and preparing staff from unaffected districts. However, managing the available human resources to staff cholera treatment centres, mobiliszing response teams to affected rural areas and maintaining routine services in rural facilities was complicated and difficult.

Stakeholders felt the success of the strategy was due to the strong provincial leadership and the initial training of a core group of clinical staff largely by MSF once the outbreak had spread to the capital of the first affected province. The initial treatment centre established by MSF enabled clinical management training of many health care workers who had never been exposed to cholera and provided a platform for operational research. ${ }^{8}$ The training ensured the nurse unit managers and other clinical staff were competent not only in effectively managing cholera cases but also in running a treatment centre. Operating the centre involved activities such as rostering, clinical audits, ongoing training, cleaning, provision of water and sanitation, procurement, infection control and mortuary services. The strategy was less effective in locations where existing leadership was weak. In such locations, stakeholder technical assistance was rejected, financial resource allocation did not correspond to interventions prioritized by the provincial task force, and the rotated district staff did not always perform the activities they were recruited to perform.

During the later stages of the outbreak, experienced cholera treatment centre teams from the first-affected provinces were recruited to work in cholera treatment centres in other provinces with high mortality. This was effective for improving the management of cases and the treatment centre during the period the team was on the ground. However, it did little to improve the situation in the longer term as opportunities to share the expertise of clinical staff from previously affected provinces were not seized and not much was done to improve systems. Staff rotation also enabled clinical staff to witness their colleagues treating cases of this "new disease" and not getting sick or dying. In this way, experienced staff were able to reassure colleagues who had fled their health facilities for fear of the disease. Cholera outbreaks, like the one in Papua New Guinea, can be expensive if they continue for months in settings with limited infrastructure. The cholera treatment centres were initially staffed with clinicians, infection control officers and security guards at an overall cost of approximately US $\$ 13500$ per month (largely for salaries).

\section{(11) Rosters and volunteers are essential for staff rotation in remote settings}

In settings where health care workers were present, unpaid volunteers often provided safe water to the makeshift treatment centres, monitored intravenous flask needs of patients in their homes before moving to makeshift treatment facilities and prepared rehydration solutions for patients as well as chlorine solutions for infection control. Such activities enabled health care workers to catch up on much-needed sleep during intense periods of transmission in remote areas. To complement the important contributions of volunteers, district health authorities developed rosters of staff from nearby facilities to ensure that clinicians shared shifts with community health workers during periods of intense transmission. In settings where strong local leadership was absent, a functional roster system was a challenge and staff were frequently overburdened, placing patient lives at risk.

\section{DISCUSSION}

Clinicians are the backbone of primary health care in Papua New Guinea and include mostly community health workers and health extension officers with far fewer nurses and doctors. They are essential in the implementation of mortality-reduction interventions during outbreaks and for ensuring the ongoing function of essential health services. For these reasons, mapping and projecting population health needs for clinical staff has been prioritized in Papua New Guinea. However, a variety of public health professionals are required for health emergency planning, preparedness and response. They include officers trained in environmental health, health promotion, logistics, communications, laboratory diagnosis and surveillance, data management, field epidemiology as well as monitoring and evaluation. The creation of a cadre of trained field epidemiologists to monitor disease trends, provide intelligence to those 
conducting risk assessments, inform decision-makers about potential disease threats and guide the response during a public health emergency is essential. Objectives of the national health emergencies plan are best achieved if the required human resources are clearly identified and articulated in the national human resources plan.

The Papua New Guinea response to cholera demonstrates system inadequacies, including the systems that identify, develop and make projections on human resource requirements for health. Generally, locations that functioned well before the epidemic responded better to it, especially in provinces where emergency funding arrangements had been put in place before the outbreak. Pre-service training, opportunities for ongoing training, increased supervisory visits, production, utilization of all public health professionals, supervision and support, financial support and incentives, housing and training in supervision and outreach have all been previously identified as areas for strengthening. ${ }^{9}$ Developing human resource targets is important for achieving desired health system outcomes. The target of at least one trained field epidemiologist per 200000 population is an example of a benchmark that should be established for Papua New Guinea. ${ }^{10}$ However, there are several other cadres of public health professionals that are required for emergency response, all of which are currently in limited supply and would also benefit from such targets. These cadres should also feature in the mapping and projections of public health professional needs in any fully adopted national workforce plan and may be a consideration for targets relating to their ratio to the population.

\section{CONCLUSION}

The human resources for health in Papua New Guinea made emergency response a challenge during the cholera outbreak. While the outbreak response was generally well managed, improvement to human resource systems before the next emergency will enable a more effective response as is essential for the achievement of the IHR core capacity requirements. Public health professionals needed for health emergency preparedness and response should be mapped, and planning should be implemented to increase the numbers in relevant areas.
Human resource planning should be integrated with health emergency planning. It is essential to maintain and strengthen the human resource capacities and experiences gained during the cholera outbreak to ensure a more effective response to the next health emergency.

\section{Conflicts of interest}

None declared.

Funding

None.

\section{References:}

1. International Health Regulations (2005), Second Edition. Geneva, World Health Organization, 2008 (http://whqlibdoc. who.int/publications/2008/9789241580410_eng.pdf, accessed 15 April 2013).

2. Asia Pacific Strategy for Emerging Diseases (2010). Manila, World Health Organization Regional Office for the Western Pacific, 2011 (http://www.wpro.who.int/emerging_diseases/APSED2010/ en/index.html, accessed 15 April 2013).

3. Countries - Papua New Guinea. Geneva, World Health Organization, 2013 (http://www.who.int/countries/png/en/, accessed 15 April 2013).

4. Papua New Guinea health workforce crisis: a call to action. Washington, DC, The World Bank, 2011 (http://www-wds. worldbank.org/external/default/WDSContentServer/WDSP/IB/2 013/01/24/000333037_20130124103924/Rendered/PDF/ NonAsciiFileName0.pdf, accessed 15 April 2013).

5. Dawson H, Gray K. Human resources for health in maternal, neonatal and reproductive health at community level: A profile of Papua New Guinea. Sydney, University of New South Wales, 2011 (http://www.hrhhub.unsw.edu.au/HRHweb.nsf/resources/ MNRH PNG-Web.pdf/\$file/MNRH PNG-Web.pdf, accessed 15 April 2013).

6. Rosewell A et al. Vibrio cholerae 01 in 2 coastal villages, Papua New Guinea. Emerging Infectious Diseases, 2011, 17:154-156. doi:10.3201/eid1701.100993 pmid:21192890

7. Horwood PF et al. Clonal origins of Vibrio cholerae $01 \mathrm{El}$ Tor strains, Papua New Guinea, 2009-2011. Emerging Infectious Diseases, 2011, 17:2063-2065. doi:10.3201/eid1711.110782 pmid:22099099

8. Rosewell A et al. Cholera risk factors, Papua New Guinea, 2010. BMC Infectious Diseases, 2012, 12:287. doi:10.1186/14712334-12-287 pmid:23126504

9. Foster M et al. Working Paper 1: Papua New Guinea Country Report. Canberra: Australian Agency for International Development, 2009 (http://www.ode.ausaid.gov.au/publications/ documents/working-paper-health-service-delivery-png.pdf, accessed 15 April 2013).

10. Ijaz K et al. International Health Regulations-what gets measured gets done. Emerging Infectious Diseases, 2012, 18:1054-1057. doi:10.3201/eid1807.120487 pmid:2270959 
